\title{
HSPICE Macromodel of a Programmable Metallization Cell (PMC) and its Application to Memory Design
}

\author{
Pilin Junsangsri, Fabrizio Lombardi \\ Department of Electrical and Computer Engineering \\ Northeastern University \\ Boston, MA USA 02115 \\ junsangsri.p@husky.neu.edu, lombardi@ece.neu.edu
}

\author{
Jie Han \\ Department of Electrical and Computer Engineering \\ University of Alberta \\ Edmonton, Canada \\ jhan8@ualberta.ca
}

\begin{abstract}
This paper presents a new HSPICE macromodel of a Programmable Metallization Cell (PMC). The electrical characteristics of a PMC are simulated by using a geometric model that considers the vertical and lateral growth/dissolution of the metallic filament. The selection of the parameters is based on operational features, so the electrical characterization of the PMC is simple, easy to simulate and intuitive. The I-V and R-V plots of a PMC are generated at a very small error compared with experimental data; the proposed model also shows a small error for the relationship between the switching time and the pulse amplitude. The use of a PMC as resistive element in a crossbar memory is also presented; it is shown that a PMC-based crossbar offers substantial improvements over other resistive technologies.
\end{abstract}

Keywords-Conducting Bridge Random Access Memory (CBRAM), Programmable Metallization Cell (PMC), HSPICE, Modeling, Emerging Technology.

\section{INTRODUCTION}

The Programmable Metallization Cell (PMC), also known as the Conducting Bridge Random Access Memory (CBRAM) or solid-electrolyte memory, is a device technology that uses the phenomenon of resistive switching in design [1]. The PMC has excellent speed $(<10 \mathrm{~ns})$, scalability to a sub-22-nm regime, extremely low power consumption (in nano watts), good retention and endurance [1]. Hence, PMC is a good candidate for the next generation of non-volatile memories.

However, simulation tools that are compatible with CMOS based design environments (such as HSPICE) are needed to integrate a PMC into an IC. In this paper, the HSPICE macromodel of a programmable metallization cell (PMC) is initially proposed. The proposed PMC macromodel is versatile and comprehensive. The electrical characteristic of the PMC are generated by utilizing a geometry-based model that considers the vertical and lateral growth/dissolution of the metallic filament. This paper has also shown that different from other models found in the technical literature [1] [2], the switching time and voltage of the proposed macromodel are interrelated as well as with the voltage drop across the PMC. The I-V and R-V plots and the relationship between the switching time and the pulse amplitude of the PMC are modeled at a very small error compared with experimental data. The application of a PMC as a nonvolatile element in a crossbar memory is presented. A comparison with crossbars utilizing MOSFETs and memristors as switches shows that the PMC-based crossbar provides significant performance advantages.

\section{Programmable Metallization Cell (PMC)}

The Programmable Metallization Cell (PMC) is a resistive switching memory element based on the migration of metallic ions through a solid electrolyte and the subsequent formation and dissolution of a metallic conductive filament (CF) connecting the two electrodes $[3,4]$.

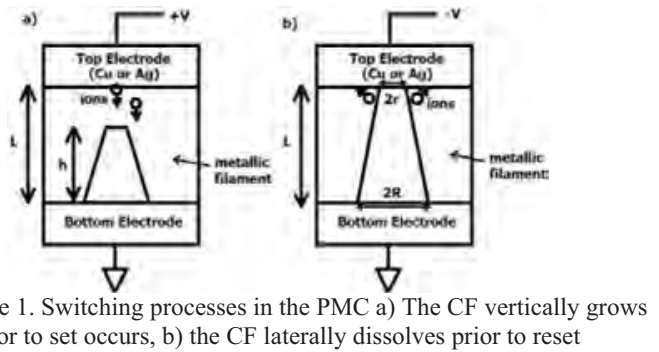

The set (OFF to ON state transition) and the reset (ON to OFF state transition) processes of a PMC device are shown in Figure 1.

- Under a positive bias, the top active electrode is oxidized, and the fast metal ions $\left(\mathrm{Ag}^{+}\right.$or $\left.\mathrm{Cu}^{2+}\right)$ drift toward the bottom electrode and form the $\mathrm{CF}$. Thus, the $\mathrm{CF}$ vertically grows until it reaches the top electrode, at which time the set occurs. Following the set, the CF grows laterally and its diameter continues to increase, because more metal ions are present around it $[1,3]$.

- For the reset process, a negative voltage bias occurs across the PMC (Figure 1b); the CF tends to laterally 
dissolve, because the enhanced lateral electric field is at the top of the CF [5]. The reset process is completed when the diameter of the conductive filament shrinks down to zero at the top electrode. After the reset, the CF vertically dissolves and its height keeps decreasing.

The switching process of a PMC has a transition point that occurs whenever the tip of the $\mathrm{CF}$ touches or separates from the top electrode. The OFF state occurs when the tip of the conductive filament is separated from the top electrode; so, the $\mathrm{CF}$ height (h) is less than the film thickness of the solid electrolyte or the height of the PMC (L). The ON-state resistance of a PMC $\left(\mathrm{R}_{\mathrm{on}}\right)$ occurs when the tip of CF touches the top electrode. The OFF and ON state resistances of the $\mathrm{PMC}\left(\mathrm{R}_{\mathrm{off}}\right.$ and $\left.\mathrm{R}_{\mathrm{on}}\right)$ are found by using the following equations $[1]$.

$$
\begin{gathered}
\mathrm{R}_{\text {off }}=\left(\rho_{\text {on }} \mathrm{h}+\rho_{\text {off }}(\mathrm{L}-\mathrm{h})\right) / \mathrm{A} \\
\mathrm{R}_{\text {on }}=\rho_{\text {on }} \mathrm{L} /(\pi \mathrm{rR})
\end{gathered}
$$

where $\rho_{\text {on }}$ is the CF resistivity, $\rho_{\text {off }}$ is the non-conducting solid-electrolyte resistivity, $\mathrm{L}$ is the film thickness of the solid electrolyte and A is the area at the bottom of the CF (on the assumption that it is cylindrical before the set process). $\mathrm{R}$ is the radius at the bottom of the $\mathrm{CF}$ and $\mathrm{r}$ denotes the $\mathrm{CF}$ radius.

The values of $h$ and $r$ vary based on time and bias voltage across the PMC cell, the evolution rates of the CF height and radius are given by [1].

$$
\begin{aligned}
& \mathrm{dh} / \mathrm{dt}=\mathrm{v}_{\mathrm{h}}{ }^{*} \exp \left(-\mathrm{E}_{\mathrm{a}} / \mathrm{kT}\right) * \sinh (\alpha \mathrm{V} / \mathrm{kT}) \\
& \mathrm{dr} / \mathrm{dt}=\mathrm{v}_{\mathrm{r}}{ }^{*} \exp \left(-\mathrm{E}_{\mathrm{a}} / \mathrm{kT}\right) * \sinh (\beta \mathrm{V} / \mathrm{kT})
\end{aligned}
$$

where $\alpha$ is a fitting parameter [1], $\mathrm{E}_{\mathrm{a}}$ is the activation energy, $\mathrm{kT}$ is the thermal energy, $\mathrm{v}_{\mathrm{h}}$ is the $\mathrm{CF}$ vertical growth velocity, $r$ is the radius of $C F$ at the top of the filament, $v_{r}$ and $\beta$ are the fitting parameters for the evolution velocity and the electric field dependence respectively, and $\mathrm{V}$ is the bias voltage across the PMC [1]. $\mathrm{h}$ and $\mathrm{r}$ are found based on the evolution rates of (3) and (4) and the PMC resistance is calculated by using (1) and (2).

\section{PROPOSED MACROMOdEL}

A new HSPICE macromodel of a PMC is proposed in this manuscript. The $\mathrm{CF}$ height (h), the $\mathrm{CF}$ radius (r) and the state of the PMC are found when considering the CF volume; hence it is a geometry-based model. The proposed PMC macromodel has two terminals, in and out: in is the input terminal, while out is the output terminal.

a)

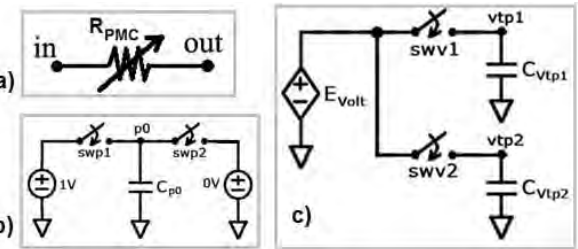

Figure 2. a) Circuit model of programmable metallization cell (PMC) b) Voltage polarity checking circuit c) Previous CF volume storing circuit

Figure 2a) presents the circuit model of a PMC, basically it is a variable resistor. The $\mathrm{PMC}$ resistance is given by

$$
\mathrm{R}_{\mathrm{PMC}}=\mathrm{R}_{\text {off }} * \mathrm{~V}_{\text {Coff }}+\mathrm{R}_{\mathrm{on}} *\left(1-\mathrm{V}_{\text {Coff }}\right)
$$

where $V_{\text {Coff }}$ represents the state of the PMC, i.e. if the PMC is in the OFF (ON) state, then $V_{\text {Coff }}$ is $1(0)$. $R_{\text {off }}$ and $R_{\text {on }}$ are the
$\mathrm{OFF}$ and the ON-state resistances of the PMC (given previously in (1) and (2) respectively). Since the OFF and ONstate resistances of the PMC are based on its CF height (h) and radius (r), the largest $\mathrm{CF}$ height $\left(\mathrm{h}_{\mathrm{th}}\right)$ and the least $\mathrm{CF}$ radius $\left(\mathrm{r}_{\text {th }}\right)$ must be calibrated to ensure that the resistance of the PMC is continuous when changed. The maximum CF height $\left(\mathrm{h}_{\mathrm{th}}\right)$ is selected at the value close to the height of the PMC (L), while the value of the least $\mathrm{CF}$ radius $\left(\mathrm{r}_{\mathrm{th}}\right)$ is found from (6).

$$
\mathrm{r}_{\text {th }}=\rho_{\text {on }} \mathrm{L} /\left(\pi \mathrm{R}\left(\left(\rho_{\mathrm{on}} \mathrm{h}_{\mathrm{th}}+\rho_{\mathrm{off}}\left(\mathrm{L}-\mathrm{h}_{\mathrm{th}}\right)\right) / \mathrm{A}\right)\right)
$$

The relationships between the switching time of the set and reset processes and the pulse amplitude are given as follows

$$
\begin{aligned}
& \mathrm{t}_{\text {set }}=\mathrm{t}_{\mathrm{p}}=\alpha^{*} \exp \left(\beta^{*} \mid \mathrm{V}_{\text {in,out }}\right) \\
& \mathrm{t}_{\mathrm{reset}}=\mathrm{t}_{\mathrm{n}}=\gamma^{*} \exp \left(\delta^{*}\left|\mathrm{~V}_{\text {in,out }}\right|\right)
\end{aligned}
$$

where $t_{\text {set }}$ or $t_{p}$ is the switching time of the set process that occurs when a positive voltage drop exists across the PMC. $t_{\text {reset }}$ or $t_{n}$ is the switching time of the reset process that occurs when a negative voltage drop exists across the PMC. Curve fitting is then utilized for the other parameters. Their values are based on experimental data; if the results of [1] are utilized, $\alpha, \beta, \gamma$, and $\delta$ are equal to $679.27,-16.73,149.97$, and -14.86 respectively.

The change in the CF volume at each time step (denoted by tstart) can be found by considering the switching time of the set and reset processes, i.e. the time that the CF of a PMC changes from an height equal to zero to a point where the $\mathrm{CF}$ radius at the top electrode increases up to a value equal to the radius at the bottom of the $\mathrm{CF}(\mathrm{R})$ (or vice versa). In this paper, the shape of the CF is assumed to be conical; it is then converted to a cylindrical form when the $\mathrm{CF}$ radius at the top electrode ( $\mathrm{r}$ ) reaches the radius at the bottom of the CF (R). As the metal ions drift toward the bottom electrode at a constant rate, then the changing rate of the $\mathrm{CF}$ volume ( $\mathrm{dVol})$ is also constant and given by

$$
\begin{array}{r}
d \text { Volp }=\frac{\pi R^{2} * \text { hth } * \text { tstart }}{t_{p}} \\
d \text { Voln }=\frac{-\pi R^{2} * \text { hth } * \text { tstart }}{t_{n}}
\end{array}
$$

(9) and (10) give the changing rates of the CF volume during the set and reset processes (positive and negative). When a positive voltage exists across a PMC, the $\mathrm{CF}$ volume is increased; however if a negative voltage exists across the $\mathrm{PMC}$, the $\mathrm{CF}$ volume decreases. The instantaneous $\mathrm{CF}$ volume can be found from the changing rate of the $\mathrm{CF}$ volume as follows.

$$
\operatorname{Vol}(\mathrm{t})=\mathrm{Vol}_{\text {prev }}+\mathrm{dVol}(\mathrm{t})+\mathrm{Vol}_{\mathrm{adj}}
$$

where $\mathrm{Vol}_{\text {prev }}$ is the $\mathrm{CF}$ volume at the previous (simulated) time step, $\mathrm{Vol}_{\mathrm{adj}}$ is the adjusted $\mathrm{CF}$ volume that is used to control the $\mathrm{CF}$ volume (i.e. between 0 and the largest value), $\mathrm{dVol}(\mathrm{t})$ is the changing rate of the $\mathrm{CF}$ volume at a specific time. Its value is given by

$$
\mathrm{dVol}(\mathrm{t})=\mathrm{dVolp} * \mathrm{~V}_{\mathrm{p} 0}+\mathrm{dVoln} *\left(1-\mathrm{V}_{\mathrm{p} 0}\right)
$$

where $\mathrm{V}_{\mathrm{p} 0}$ is the voltage at node $\mathrm{p} 0$ of the voltage polarity checking circuit (Figure 2b)). This circuit is used to check the polarity of the voltage difference across the PMC.

- If a positive voltage is dropped across the PMC, switch swp1 is ON, while switch swp2 is OFF, the voltage at node $\mathrm{p} 0$ is $1 \mathrm{~V}$. 
- If the voltage difference across the PMC is negative, switches swp1 and swp2 are OFF and ON respectively, the voltage at node $\mathrm{p} 0$ is zero

The CF volume at the previous time step $\left(\mathrm{Vol}_{\text {prev }}\right)$ is simulated in the proposed macromodel using the circuit in Figure 2c). The voltage source $E_{\text {volt }}$ generates the instantaneous CF Volume (in (11)), while the initial voltages at nodes vtp1 and vtp2 are given by the initial CF volume of the PMC $\left(\mathrm{Vol}_{\text {ini }}\right)$. Two capacitors are employed to store the value of the $\mathrm{CF}$ volume at the previous time step ( $\left.\mathrm{Vol}_{\text {prev }}\right)$. $\mathrm{Vol}_{\text {prev }}$ is found by generating a voltage pulse whose value changes at every time step. This is accomplished as follows.

- Switches swv1 and swv2 are ON when the voltage pulse is equal to 0 and 1 respectively.

- The instantaneous CF volume of the PMC is stored at vtp1 and vtp2 based on the value of the voltage pulse (the previous $\mathrm{CF}$ volume is found from different nodes).

- If the voltage pulse is 1 , the CF volume at the previous time step is equal to the voltage at vtp1.

- If the voltage pulse is zero, the previous $\mathrm{CF}$ volume of the PMC is found from the voltage at vtp2. So,

$$
\mathrm{Vol}_{\text {prev }}=\mathrm{V}_{\mathrm{VTP} 1} * \mathrm{~V}_{\text {pulse }}+\mathrm{V}_{\mathrm{VTP} 2} *\left(1-\mathrm{V}_{\text {pulse }}\right)
$$

where $\mathrm{V}_{\text {pulse }}$ is the pulse voltage that is generated to control switches swv1 and swv2.

After finding $\mathrm{Vol}_{\text {prev }}$ and $\mathrm{dVol}(\mathrm{t})$, the adjusted $\mathrm{CF}$ volume $\mathrm{Vol}_{\text {adj }}$ is considered (based on (11)) to ensure that the $\mathrm{CF}$ volume remains in range.

- When the CF volume is larger than the largest value, $\mathrm{Vol}_{\mathrm{adj}}$ is given by the difference between instantaneous $\mathrm{CF}$ volume $\left(\mathrm{Vol}_{\text {prev }}+\mathrm{dVol}(\mathrm{t})\right.$, or $\left.\mathrm{V}_{\text {vols }}\right)$ and its largest value $\left(\mathrm{V}_{\mathrm{Vols}}-\mathrm{Vol}_{\max }\right)$.

- If the CF volume is negative, the adjusted CF volume is given by a value that is equal to the instantaneous $\mathrm{CF}$ volume (i.e. $\mathrm{V}_{\text {Vols }}$ ).

Hence,

$$
\mathrm{Vol}_{\mathrm{adj}}=\mathrm{V}_{\mathrm{adjH}} *\left(\mathrm{~V}_{\mathrm{Vols}}-\mathrm{Vol}_{\text {max }}\right)+\mathrm{V}_{\mathrm{adjL}} * \mathrm{~V}_{\mathrm{Vols}}
$$

where $\mathrm{V}_{\mathrm{Vols}}$ is the instantaneous CF volume; it is equal to the sum of the previous $\mathrm{CF}$ volume and the changing rate of the $\mathrm{CF}$ volume at each time step $\left(\mathrm{Vol}_{\text {prev }}+\mathrm{dVol}(\mathrm{t})\right), \mathrm{Vol}_{\max }$ is the largest allowed $\mathrm{CF}$ volume, $\mathrm{V}_{\mathrm{adjH}}\left(\mathrm{V}_{\mathrm{adjL}}\right)$ is the voltage to control the adjusted $\mathrm{CF}$ volume, it is equal to 1 when $\mathrm{V}_{\text {Vols }}-$ $\mathrm{Vol}_{\max }$ is positive ( $\mathrm{V}_{\mathrm{Vols}}$ is negative); otherwise, it is set to zero. Therefore, the instantaneous CF volume of the PMC $(\operatorname{Vol}(\mathrm{t}))$ is given by $(11)$.

After finding the CF volume, the state of the PMC (OFF or $\mathrm{ON}$ ) can be established. As the shape of the CF is conical, the largest $\mathrm{CF}$ volume in the $\mathrm{OFF}$ state is equal to the volume of the cone when its height is at the largest value $\left(\mathrm{h}_{\mathrm{th}}\right)$. This is also referred to as the threshold volume of the $\mathrm{CF}\left(\mathrm{Vol}_{\mathrm{th}}\right)$. If the $\mathrm{CF}$ volume is larger than its threshold value, the PMC is switched to the ON state. (15) gives the threshold volume of the $\mathrm{CF}$ as used for the state of the PMC.

$$
\mathrm{Vol}_{\mathrm{th}}=\frac{1}{3} \pi \mathrm{R}^{2} \mathrm{~h}_{\mathrm{th}}
$$

Next, the CF height and radius of the PMC are found. In the OFF state, the shape of the $\mathrm{CF}$ is conical and its volume is given by (16), while the CF height is given by (17)

$$
\operatorname{Vol}(\mathrm{t})=\frac{1}{3} \pi R^{2} h
$$

$$
\mathrm{h}=\frac{\mathrm{Vol}(\mathrm{t}) * 3}{\pi \mathrm{R}^{2}}
$$

where $\mathrm{h}$ is the instantaneous $\mathrm{CF}$ height whose value is bound between 0 and the largest $\mathrm{CF}$ height $\left(\mathrm{h}_{\mathrm{th}}\right)$.

However if the $\mathrm{PMC}$ is in the $\mathrm{ON}$ state, the $\mathrm{CF}$ radius (r) must be also considered. In the $\mathrm{ON}$ state, the $\mathrm{CF}$ shape is in frustum cone form whose volume is given by (18). The CF radius is found in (19).

$$
\begin{gathered}
\operatorname{Vol}(\mathrm{t})=\frac{\pi \mathrm{h}}{3}\left(\mathrm{R}^{2}+\mathrm{Rr}+\mathrm{r}^{2}\right) \\
\mathrm{r}=\frac{\sqrt{3\left(4 \pi \mathrm{h}_{\mathrm{th}} \operatorname{Vol}(\mathrm{t})-\mathrm{h}_{\mathrm{th}}{ }^{2} \pi^{2} \mathrm{R}^{2}\right)}-\pi R h_{\mathrm{th}}}{2 \pi \mathrm{h}_{\mathrm{th}}}
\end{gathered}
$$

where the $\mathrm{CF}$ radius has its least and largest values at $r_{\text {th }}$ and $\mathrm{R}$ respectively. After the $\mathrm{CF}$ height and radius are found, the resistance is calculated using (1) and (2) respectively. Finally, the electrical characteristics of the PMC can be simulated.

Based on (7) and (8), the switching time is still constant when the size of the PMC varies; this is however incorrect. Since the relationship between the switching time and the PMC size is not precisely known and for simplicity of analysis the switching time is made to be linearly dependent with the $\mathrm{CF}$ volume. Therefore, the relationships between switching time of the set and reset processes and the pulse amplitude of the PMC are given by

$$
\begin{aligned}
& \mathrm{t}_{\text {set }}=\mathrm{t}_{\mathrm{p}}=\alpha \exp \left(\beta^{*}\left|\mathrm{~V}_{\mathrm{in}, \text { out }}\right|\right) * \frac{\mathrm{Vol}_{\text {max }}}{\mathrm{Vol}_{\mathrm{de}}} \\
& \mathrm{t}_{\text {reset }}=\mathrm{t}_{\mathrm{n}}=\gamma \exp \left(\delta^{*}\left|\mathrm{~V}_{\text {in,out }}\right|\right) * \frac{\mathrm{Vol}_{\text {max }}}{\mathrm{Vol}_{\text {de }}}
\end{aligned}
$$

where $\mathrm{Vol}_{\max }$ is the largest CF volume (equal to $\pi \mathrm{R}^{2} \mathrm{~h}_{\mathrm{th}}$ ), $\mathrm{Vol}_{\mathrm{de}}$ is the default value of the $\mathrm{CF}$ volume. This is found using experimental data, i.e. if [1] is used, the CF height is $49.5 \mathrm{~nm}$ and the CF radius is $20.57114 \mathrm{~nm}$.

So, the changing rate of the $\mathrm{CF}$ volume at each time step is related to the voltage difference across the PMC; the voltage difference across the PMC (that is used to calculate the switching time in (20) and (21)) is between the least and largest voltage values (to limit the CF volume within the range of 0 and $\left(\mathrm{Vol}_{\max }\right)$. The largest and least voltage differences across the PMC are given in (20) and (21) respectively and $t_{p}$ and $t_{n}$ are equal to tstart.

$$
\begin{aligned}
& \mathrm{V}_{\text {PMC,Max }}=-\frac{1}{\beta} \ln \left(\frac{\mathrm{t}_{\text {start }} \mathrm{Vol}_{\text {de }}}{\text { Vol }_{\text {max }^{* \alpha}}}\right) \\
& \mathrm{V}_{\mathrm{PMC}, \text { Min }}=-\frac{1}{\delta} \ln \left(\frac{\mathrm{t}_{\mathrm{start} \mathrm{Vol}_{\mathrm{de}}}}{\mathrm{Vol}_{\max } \gamma}\right)
\end{aligned}
$$

\section{Simulation Results}

In this section, the proposed HSPICE macromodel of a PMC is assessed; the data of [1] (shown in Table 1) is initially utilized for the physical parameters. The electrical characteristics of a PMC are simulated and assessed as follows.

Table 1. Parameters used in simulation (from [1])

\begin{tabular}{|c|c|c|c|}
\hline Parameters & Value & Parameter & Value \\
\hline$\rho_{\text {on }}(\Omega \bullet n m)$ & $4 * 10^{7}$ & $\mathrm{R}(\mathrm{nm})$ & 20.57114 \\
\hline$\rho_{\mathrm{off}}(\Omega \bullet \mathrm{nm})$ & $1.33^{*} 10^{11}$ & $\alpha$ & 679.27 \\
\hline $\mathrm{h}_{\mathrm{th}}(\mathrm{nm})$ & 49.5 & $\beta$ & -16.73 \\
\hline $\mathrm{r}_{\text {th }}(\mathrm{nm})$ & 0.75 & $\gamma$ & 149.97 \\
\hline $\mathrm{A}\left(\mathrm{nm}^{2}\right)$ & 1330 & $\delta$ & -14.86 \\
\hline
\end{tabular}




\section{A. CF height and radius}

The variation of the $\mathrm{CF}$ height $(\mathrm{h})$ and radius (r) must be considered to evaluate the electrical characteristics of a PMC; they are shown in Figures $3 \mathrm{~b}$ and $3 \mathrm{c}$ respectively by utilizing the voltage pulse sequence of Figure $3 \mathrm{a}$.
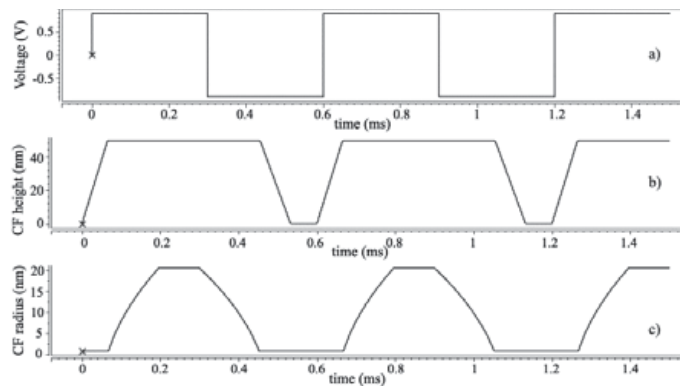

Figure 3. a) Voltage pulse sequence across PMC b) CF height c) CF radius of PMC Vs Simulation time (ms)

The CF height increases (Figure 3 ) when a positive voltage is dropped across the PMC. The CF radius is found when the $\mathrm{CF}$ height reaches its largest value. However, if a negative voltage is dropped across the $\mathrm{PMC}$, the $\mathrm{CF}$ radius is reduced; when the $\mathrm{CF}$ radius is reduced to its least value the tip of the $\mathrm{CF}$ is separated from the top electrode and the CF height decreases. These characteristics are well matched with the variations of $\mathrm{CF}$ height and radius (Figure 3).

\section{B. I-V and R-V Plots}

The I-V and R-V plots of a PMC are generated next. These are found by simulating the DC double sweep of Figure 4a.

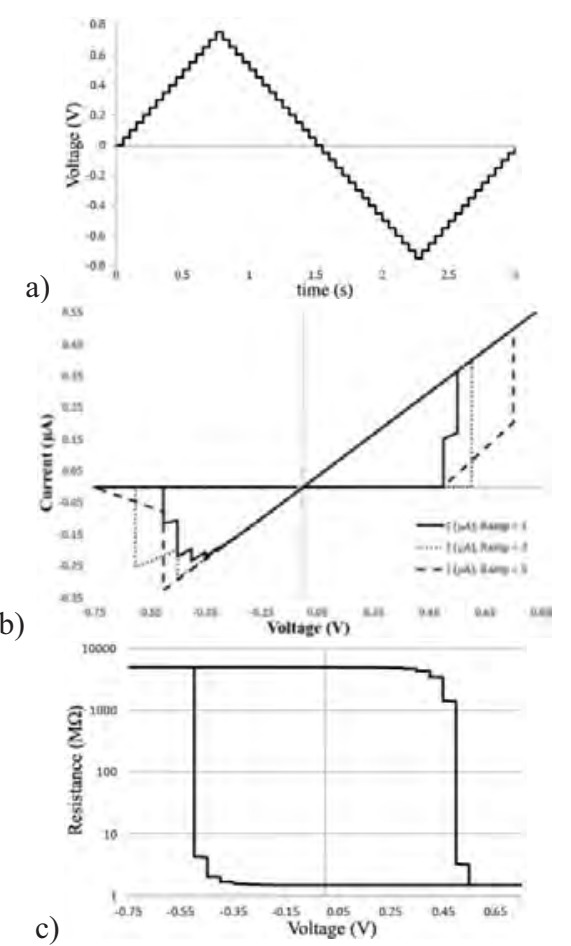

Figure 4. a) Voltage difference across PMC for generating I-V and R-V plots b) I-V characteristics of the proposed PMC macromodel when the ramp rate of the DC Sweep is 1,3 and $5 \mathrm{~V} / \mathrm{s} \mathrm{c}$ ) R-V characteristics of the proposed PMC macromodel
Figures $4 \mathrm{~b}$ and $4 \mathrm{c}$ present the $\mathrm{I}-\mathrm{V}$ and $\mathrm{R}-\mathrm{V}$ characteristics of the proposed PMC macromodel when the voltage difference across the PMC is swept (Figure 4a). The initial CF height starts at $0 \mathrm{~nm}$; as a positive voltage drop occurs across the PMC, the CF height increases (and its increasing rate is dependent on the voltage difference). If the voltage difference across the PMC is high, then the changing rates of the $\mathrm{CF}$ height and radius are also high and the PMC is suddenly switched to the ON state. The reset process is similar to the set process; when the negative voltage difference across the PMC is high, the changing rates of the $\mathrm{CF}$ height and radius are also high. Therefore, the PMC is suddenly switched to the OFF state. The I-V and R-V curves (Figures $4 \mathrm{~b}$ and $4 \mathrm{c}$ respectively) clearly show these characteristics.

- When the positive voltage difference across the PMC is larger than $0.5 \mathrm{~V}$, the changing rate of the $\mathrm{CF}$ height and radius are high. So, the PMC is suddenly switched to the ON state (low resistance).

- When the negative voltage difference across the PMC is larger than $0.35 \mathrm{~V}$, the PMC is suddenly switched to the OFF state (high resistance).

\section{Switching Times}

A comparison between the switching times of the set and reset processes using the proposed macromodel and the experimental results [1] is pursued.

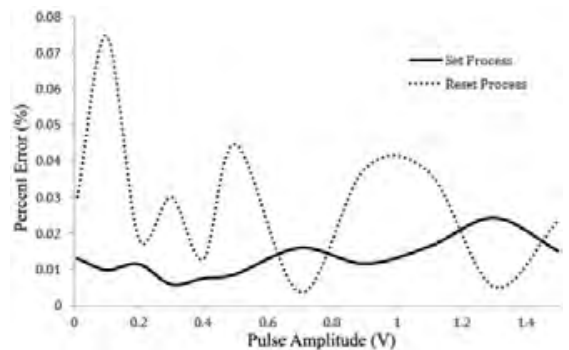

Figure 5. Percentage errors between the switching time of the proposed PMC macromodel and the experimental results [1] for the set and reset processes vs pulse amplitude (V)

Figure 5 shows the percentage errors of the switching time for both the set and reset processes of the proposed PMC macromodel and the experimental results [1] versus pulse amplitude. The proposed PMC macromodel simulates the switching time for both processes very closely to the experimental data of [1], i.e. at a largest error less than $0.08 \%$.

\section{Set Voltage and Ramp Rate}

The relationship between the set voltage and the ramp rate of the proposed PMC macromodel is established. The ramp rate of the DC sweep (Figure 4a) is defined as the voltage step divided by the duration time; so, when the ramp rate is increased, the set voltage also increases.

Figure $4 \mathrm{~b}$ ) presents the I-V characteristics of the proposed PMC macromodel when the ramp rate of the double DC sweep is changed. When the ramp rate is increased, the set voltage of the proposed PMC macromodel also increases. This is similar to the experimental results of [1]. 


\section{APPlication: CROSSBAR MEMORY}

The crossbar memory is a memory array made of horizontal and vertical conducting wires; a switch is placed at each wire crossing. A switch of a crossbar array has two distinct states, the $\mathrm{ON}$ and the OFF states, corresponding to the low and the high resistance values respectively [6]. Each switch acts as a memory element and is programmed by a sufficiently high voltage pulse on the corresponding word and bit lines; its state is read by sensing either the corresponding voltage, or current [7].

Molecular switches have been proposed as memory elements of a crossbar array [6]. [7] has shown that by substituting molecular switches with CNTFETs, a crossbar array shows improvements in both the sense voltage on/off ratio and the noise margin compared with a molecular-based implementation. A PMC is investigated next as a switching element in a crossbar. The PMC is then assessed in this section due to ease of fabrication and the large on/off resistance ratio. Two read-out schemes are considered for such implementation.

\section{A. Read-Out Schemes}

The first read out scheme is shown in Figure 6a); the supply voltage is biased to the selected switch and the current that flows from the selected switching element to ground, is then measured [6]. A resistor (with a value given by Rsense) is connected to the selected column; the current of a column is detected, while the voltage drop across the resistor (Vsense) is compared with a reference voltage (Vref) to determine the state of the selected switch. All other switches on the unselected bit and word lines are connected to ground.

a)

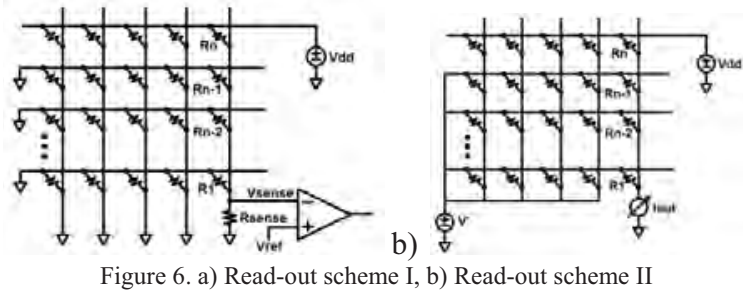

As analyzed in [6], the sense voltage of a crossbar memory is affected only by the resistances connected to the same bitlines of the selected switch. A larger array size results in a smaller absolute value of the sense voltage. Moreover, when there is a large number of $\mathrm{ON}$ switches in the array, the sense voltage is also smaller. Therefore, switches with a high $\mathrm{R}_{\mathrm{ON}} / \mathrm{R}_{\mathrm{OFF}}$ ratio are best for larger memory arrays [7]. For a $\mathrm{n} \mathrm{x}$ $\mathrm{n}$ array, the worst case scenario occurs when the state of $\mathrm{Rn}$ (Figure 6a)) is read and all other switches on the same column are ON. Let the sense voltage on/off ratio be defined as the sense voltage ratio between the ON and OFF states for the selected switch while all other switches in the array are ON. The read-out margin of the crossbar can then be found using the sense voltage on/off ratio.

The second read-out scheme is shown in Figure 6b). All unselected bitlines and/or the wordlines [7] are biased to the voltage source $\mathrm{V}^{\prime}$, while an ideal current measurement is assumed for the selected column, i.e. by measuring the current on a zero-resistance meter between the crossbar and ground instead of the voltage drop across the sense resistor.

For this read-out scheme, the relative noise margin of the sense current is considered as figure of merit [6]. The ON state current $\left(\mathrm{I}_{\mathrm{ON}}\right)$ is the sense current when the selected switch is in the ON state and the other switches are in the OFF state. Equivalently, the OFF state current $\left(\mathrm{I}_{\mathrm{OFF}}\right)$ is the sense current when all switches are in the OFF state. Therefore, the noise margin of the sense current is given by.

$$
\text { Noise Margin }=\frac{\mathrm{I}_{\mathrm{ON}}-\mathrm{I}_{\mathrm{OFF}}}{2\left(\mathrm{I}_{\mathrm{ON}}+\mathrm{I}_{\mathrm{OFF}}\right)}
$$

\section{B. Simulation results}

In this section, a 100x100 PMC-based crossbar is initially evaluated and compared with a MOSFET-based crossbar array [7]. The CF height (L) and the CF radius (R) of the PMC are $3 \mathrm{~nm}$ and $15 \mathrm{~nm}$ respectively (the values of $h_{t h}$ and $r_{\text {th }}$ are $2.975 \mathrm{~nm}$ and $0.5226481 \mathrm{~nm}$ respectively). The ON state resistance of the PMC $\left(\mathrm{R}_{\mathrm{ON}}\right)$ is now given by $169.697 \mathrm{k} \Omega$, while the OFF state resistance of the PMC $\left(\mathrm{R}_{\mathrm{OFF}}\right)$ is $564.2424 \mathrm{Meg} \Omega$. In this paper, a $32 \mathrm{~nm}$ NMOS transistor is employed as a switch and its $\mathrm{ON}$ and $\mathrm{OFF}$ states are determined by the gate voltage (at $\mathrm{V}_{\mathrm{DD}}$ and GND respectively). At this feature size, it is also assumed that the metal interconnect of the crossbar is about $32 \mathrm{~nm}$ in width, with a unit resistance of $16.526 \Omega / \mu \mathrm{m}$ and a capacitance of $276.214 \mathrm{aF} / \mu \mathrm{m}[7]$.

For the first read-out scheme, a comparison is pursued between crossbar memories using PMCs, MOSFETs and memristors as switches. The sense resistance $\left(R_{\text {sense }}\right)$ of the crossbar is set at $100 \mathrm{k} \Omega$.

Table 2. Sense voltage (V) of crossbar memories

\begin{tabular}{|c|c|c|c|}
\hline Scenarios & MOSFET & PMC & Memristor \\
\hline All switches OFF & $504.1 * 10^{-6}$ & $0.156^{*} 10^{-3}$ & $7.20^{*} 10^{-3}$ \\
\hline All switches ON & $2.204 * 10^{-3}$ & $8.58^{*} 10^{-3}$ & $4.5623^{*} 10^{-3}$ \\
\hline $\begin{array}{c}\text { One switch ON, remaining } \\
\text { switches OFF }\end{array}$ & 0.3551807 & 0.329 & 0.3639 \\
\hline $\begin{array}{c}\text { One switch OFF, } \\
\text { remaining switches ON }\end{array}$ & $5.419^{*} 10^{-6}$ & $10.3 * 10^{-6}$ & $4.3948^{*} 10^{-3}$ \\
\hline $\begin{array}{c}\text { Sense voltage on/off ratio } \\
\text { (worst case scenario) }\end{array}$ & 406.8422 & 827.29 & 1.0381 \\
\hline
\end{tabular}

The highest value of the sense voltage occurs when the selected switch is in the $\mathrm{ON}$ state, while the remaining switches are in the OFF state; when the selected switch is in the ON state, its resistance is low, so the voltage drop across it is low and therefore the sense voltage of the crossbar is large. When the remaining switches are in the OFF state, no leakage is encountered; therefore, the sense voltage of the crossbar is very large. However, if the remaining switches in the crossbar are in the ON state, a significant leakage to GND exists and the sense voltage is very low. Table 2 shows also the worst case scenario of the sense voltage on/off ratio.

Consider the two scenarios when "all switches are ON" and "one switch is OFF and the remaining switches are ON". The values of the sense voltage on/off ratio for these scenarios are very different. The sense voltage on/off ratio for a PMC is significantly larger than for a MOSFET, while the sense voltage on/off ratio of a memristor is very small (due to the smaller resistance range). Hence, the memristor is not well suited to operate as a switch in a crossbar memory. A PMC 
has a very large resistance range and its sense voltage on/off ratio is very large too and significantly better than a MOSFET based crossbar, thus showing the best performance among the devices considered as switches in this manuscript. Hence, a memristor-based crossbar memory will not be further considered in the evaluation.

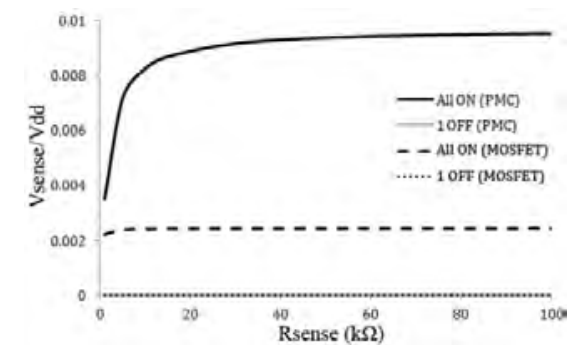

Figure 7. Ratio of sense voltage and supply voltage versus sense resistance of the crossbar

Figure 7 shows the sense voltage of the PMC and MOSFET crossbars of $100 \times 100$ size, when the sense resistance is varied under the worst case scenario; the readout margin of a PMC-based crossbar is larger than the MOSFETbased crossbar. Moreover, the sense resistance must be increased for a larger read-out margin.

Next, consider the second read-out scheme by biasing $\mathrm{V}^{\prime}$ to all unselected bitlines and/or the wordlines [7]; in this paper, the voltage $\mathrm{V}^{\prime}$ is set to $0 \mathrm{~V}$ and an ideal current measurement is utilized (i.e. at a zero resistance level for the selected bitline).

Table 3. ON/OFF current ratio of MOSFET and PMC-based crossbar memories

\begin{tabular}{|c|c|c|c|c|}
\hline \multirow{2}{*}{} & \multicolumn{2}{|c|}{ MOSFET } & \multicolumn{2}{c|}{ PMC } \\
\cline { 2 - 5 } & $\mathbf{2 0 \times 2 0}$ & $\mathbf{1 0 0 \times 1 0 0}$ & $\mathbf{2 0 \times 2 0}$ & $\mathbf{1 0 0 \times 1 0 0}$ \\
\hline ON Current (A) & $3.2034 * 10^{-5}$ & $3.1899^{*} 10^{-5}$ & $5.3029 * 10^{-6}$ & $5.3002^{*} 10^{-6}$ \\
\hline OFF Current (A) & $5.2037 * 10^{-9}$ & $5.2032^{*} 10^{-9}$ & $1.595 * 10^{-9}$ & $1.595 * 10^{-9}$ \\
\hline ON/OFF ratio & 6155.95 & 6130.57 & 3324.49 & 3323.01 \\
\hline
\end{tabular}

Table 3 presents the on/off current ratio of the MOSFET and PMC based crossbar memories; when the size of the crossbar memory is increased, the on/off current ratios of both the PMC and MOSFET-based crossbars decrease. Moreover the on/off current ratio of a MOSFET-based crossbar is larger than for the PMC-based crossbar, because a MOSFET is an active device (the $\mathrm{PMC}$ is a passive device), i.e. its $\mathrm{ON}$ current is larger than the ON current of a PMC.

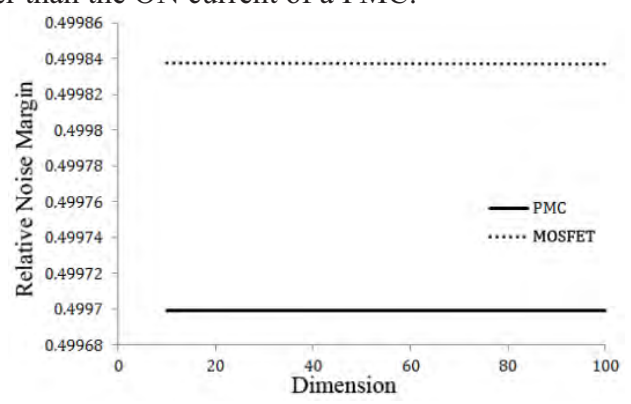

Figure 8. Relative noise margin versus crossbar dimension

Furthermore, the simulation results in Figure 8 show that when the relative noise margins of the PMC and MOSFET based crossbars are considered, an increase in array dimension has no implication as a nearly constant value is attained.

\section{CONCLUSION}

In this paper, the HSPICE macromodel of a programmable metallization cell (PMC) has been proposed. The electrical characteristics of the PMC have been generated by considering a geometry-based model such that the vertical and lateral growth/dissolution of the metallic filament has been simulated. The I-V, R-V plots and the relationship between the switching time and the pulse amplitude of the PMC have been modeled at a very small error compared with experimental data. This paper has also shown that different from other models found in the technical literature, the switching time and voltage of the proposed macromodel are interrelated as well as with the voltage drop across the PMC. The selection of the parameters in the proposed model is based on the basic operational features of a cell (such as resistance, relationship between switching time and pulse amplitude), so the electrical characterization of a PMC is simple, easily to simulate and intuitive.

Additionally, the application of a PMC as a nonvolatile element in a crossbar array has been presented. A PMC-based crossbar shows improvements in both the sense voltage on/off ratio and the read-out margin when the read-out scheme $I$ is employed. However when using the read-out scheme II, the on/off current ratio of a PMC-based crossbar is large but its value is lower than the on/off current ratio of a MOSFETbased crossbar. As for the relative noise margin, the relative noise margin of a PMC-based crossbar is close to the one for the MOSFET-based crossbar and its value is not affected by the crossbar dimension.

\section{REFERENCES}

[1] S. Yu, H.S. P. Wong "Compact Modeling of Conducting-Bridge Random-Access Memory (CBRAM)" IEEE Trans. Electron Devices, Vol. 58, No.5, May 2011

[2] D.A. Dimplu, F. Wang "Behavior Modeling of Programmable Metallization Cell Using Verilog-A" $9^{\text {th }}$ International Conference on Information Technology - New Generations 2012, IEEE Computer Society

[3] U. Russo, D. Kamalanathan, D. Ielmini, A. L. Lacaita, M. N. Kozicki "Study of Multilevel Programming in Programmable Metallization Cell (PMC) Memory" IEEE Trans. Electron Devices, Vol. 56, No.5, May 2009

[4] S. Y. Lee and K. Kim, "Future 1T1C FRAM technologies for highly reliable, high density FRAM," in IEDM Tech. Dig., 2002, pp. 547-550.

[5] X. Guo, C. Schindler, S. Menzel, and R. Waser, "Understanding the switching-off mechanism in $\mathrm{Ag}^{+}$migration based resistively switching model systems," Appl. Phys. Lett., vol. 91, no. 13, p. 133513, Sep. 2007

[6] G. Csaba, P. Lugli "Read-Out Design Rules for Molecular Crossbar Architecture" IEEE Trans. on Nanotechnology, Vol.8, No.3, May 2009 pp.369-374

[7] S. Lin, Y.B. Kim, F. Lombardi "Read-Out Schemes for a CNTFETbased Crossbar Memory” GLSVLSI $20^{\text {th }}$, pp. 167-170, May 2010 